\section{The Ergodicity Analysis of Two-Dimensional Discrete Wavelet-Based fBm Fields}

\author{
Bing-Fei Wu and Yu-Lin Su
}

\begin{abstract}
The correspondence explores the ergodicity property of the two-dimensional (2-D) discrete wavelet transform for a fractional Brownian motion (fBm) field that consists of one approximation image and three detail images. In this correspondence, the decorrelation and ergodicity of the three detail images of $\mathrm{fBm}$ fields are shown to be achieved.
\end{abstract}

Index Terms-Ergodicity, fractional Brownian motion (fBm), wavelet.

\section{INTRODUCTION}

An important problem that has appeared in the applications of stochastic processes is the estimation of various statistical parameters in terms of real data. Most parameters are expressed as the moment values, which are obtained by the ensemble averages. Fortunately, for many stationary stochastic processes, we can substitute the time averages for the unknown ensemble averages [10, p. 246], [15, p. 316]. The time average will tend to the ensemble average as the averaging interval goes to infinity when the stochastic process is ergodic. Practically, it is very difficult to check the ergodicity properties of nonstationary processes in most cases. Therefore, we usually assume that the ensemble averages of nonstationary processes may be approximated by their time averages. The fractional Brownian motion $(\mathrm{fBm})$ processes are used in a wide range of research such as $1 / f$-type noises, fractals, image textures, etc. [3], [4], [6], [9], [12], [16], where the calculation problems of the $\mathrm{fBm}$ processes are not mentioned and solved. Actually, fBm processes do not have the ergodic properties checked by the ergodicity theorem described in Papoulis [10, pp. 246-251] or Stark [15, pp. 316-322].

The ergodicity properties of the one-dimensional discrete wavelet transform (1-D DWT) of an fBm process will be stated as follows. Suppose that $B_{H}[n]$ is a sampled $\mathrm{fBm}$ process with parameter $H, 0<H<1$. From [5] and [16], the 1-D DWT of the fBm process is wide-sense stationary (WSS) and decorrelated, i.e., the autocorrelation function of the wavelet coefficient decays as $R_{d\left(B_{H}\right)}\left(m_{1}, k_{1} ; m_{2}, k_{2}\right) \sim \mathcal{O}\left(\left|2^{-m_{1}} k_{1}-2^{-m_{2} k_{2}}\right|^{2(H-M)}\right)$ for $\left|2^{-m_{1}} k_{1}-2^{-m_{2}} k_{2}\right|>\left|K_{1}+K_{2}\right|$, where $M$ is the vanishing moment of the wavelet function, the wavelet function $\psi(t)$ has a finite support given by the interval $\left[-K_{1}, K_{2}\right], K_{1} \quad$ and $\quad K_{2}>0, R_{d\left(B_{H}\right)}\left(m_{1}, k_{1} ; m_{2}, k_{2}\right) \quad \equiv$ $\mathcal{E}\left\{d_{m_{1}}\left(B_{H}\right)\left[k_{1}\right] d_{m_{2}}^{*}\left(B_{H}\right)\left[k_{2}\right]\right\}$, and $d_{m}\left(B_{H}\right)[k]$ denotes the wavelet coefficient of $B_{H}[n]$ at the resolution $m$. With these results applied to the theorem of ergodicity in [10, pp. 247-251], we conclude that the wavelet coefficient is mean ergodic. Since the $\mathrm{fBm}$ process has Gaussian distribution, the wavelet coefficient of the $\mathrm{fBm}$ is also correlation ergodic.

In this work, the results above are extended to the case of twodimensional (2-D) fBm fields. The 2-D DWT herein are based on the perfect reconstruction-quadrature mirror filter (PR-QMF) structure

Manuscript received December 13, 1996; revised September 16, 1997. This work was supported by the National Science Council under Grant NSC862213-E-009-066. The associate editor coordinating the review of this paper and approving it for publication was Dr. Phillip A. Regalia.

The authors are with the Department of Control Engineering, National Chiao Tung University, Hsinchu 300, Taiwan, R.O.C.

Publisher Item Identifier S 1053-587X(98)02017-0.
[1], [8]. The 2-D DWT includes one approximation image, which is defined as

$$
\begin{aligned}
A_{m+1} F\left[k_{x}, k_{y}\right]= & \sum_{n_{x}=-\infty}^{\infty} \sum_{n_{y}=-\infty}^{\infty} \\
& \cdot h\left[n_{x}-2 k_{x}\right] h\left[n_{y}-2 k_{y}\right] A_{m} F\left[n_{x}, n_{y}\right]
\end{aligned}
$$

and three detail images, i.e., horizontal $(\boldsymbol{H})$, vertical $(\boldsymbol{V})$, and diagonal $(\boldsymbol{D})$ detail images, which are defined as

$$
\begin{aligned}
D_{m+1}^{\boldsymbol{H}} F\left[k_{x}, k_{y}\right]= & \sum_{n_{x}=-\infty}^{\infty} \sum_{n_{y}=-\infty}^{\infty} h\left[n_{x}-2 k_{x}\right] g \\
& \cdot\left[n_{y}-2 k_{y}\right] A_{m} F\left[n_{x}, n_{y}\right], \\
D_{m+1}^{\boldsymbol{V}} F\left[k_{x}, k_{y}\right]= & \sum_{n_{x}=-\infty}^{\infty} \sum_{n_{y}=-\infty}^{\infty} g\left[n_{x}-2 k_{x}\right] h \\
& \cdot\left[n_{y}-2 k_{y}\right] A_{m} F\left[n_{x}, n_{y}\right], \text { and } \\
D_{m+1}^{\boldsymbol{D}} F\left[k_{x}, k_{y}\right]= & \sum_{n_{x}=-\infty}^{\infty} \sum_{n_{y}=-\infty}^{\infty} g\left[n_{x}-2 k_{x}\right] g \\
& \cdot\left[n_{y}-2 k_{y}\right] A_{m} F\left[n_{x}, n_{y}\right]
\end{aligned}
$$

where $m \geq 0, m, k_{x}, k_{y} \in Z, F\left[k_{x}, k_{y}\right]$ denotes a 2-D field, and $h[k]$ and $g[k]$ satisfy the QMF constraint [1], [8].

The contribution of this work is that the three detail images of 2-D fBm fields are shown to have the property of decorrelation and ergodicity that will be certified in this work. Therefore, the ensemble average can be replaced by the time average for the moment values that are presented by the numerical simulation.

The main results of this work are shown in Section II. Section III presents the numerical results. The conclusions are given in Section IV.

\section{Main Results}

\section{A. 2-D fBm Fields}

First, we summarize some properties of 2-D random fields derived from Rosenfeld [14, pp. 38-39]. Let $\boldsymbol{f}\left(\vec{r}, \omega_{i}\right)$ be a random variable, where the position vector $\vec{r}$ is given in the $x y$ plane, and $\omega_{i}$ is an outcome in the sample space. Henceforth, we will denote $\boldsymbol{f}\left(\vec{r}, \omega_{i}\right)$ by $\boldsymbol{f}(\vec{r})$ (or $\boldsymbol{f}(x, y)$ ). It is understood that this represents a family of 2-D functions with each function corresponding to an outcome $\omega_{i}$ [14, p. 39].

We wish to represent the function $\boldsymbol{f}(\vec{r})$ by its samples $\boldsymbol{f}\left(\vec{r}_{i j}\right)$ (or denoted by $f[i, j]$ ), where $\vec{r}_{i j}$ are the points on a sampling lattice as defined by

$$
\vec{r}_{i j} \equiv i \vec{r}_{1}+j \vec{r}_{2}, \quad i, j \in Z
$$

and $\vec{r}_{1}$ and $\vec{r}_{2}$ are the given basis vectors in the $x y$ plane [14, pp. 75-77]. From the definitions of mean ergodic and correlation ergodic [14, pp. 45-46] extended directly by the 1-D case, we have the following corollary such that the random field $\boldsymbol{f}\left(\vec{r}_{i j}\right)$ is mean ergodic or correlation ergodic with probability 1 .

Corollary 1: Suppose that $\boldsymbol{f}\left(\vec{r}_{i j}\right), \forall i$ and $j \in Z$ is a WSS random field with autocovariance $C_{f}\left(\vec{\tau}_{i j}\right)=R_{f}\left(\vec{\tau}_{i j}\right)-\mu_{f}^{2}$, where 


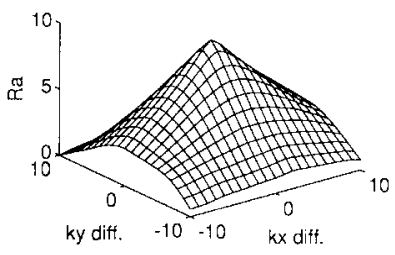

(a)

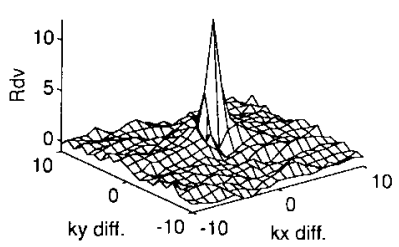

(c)

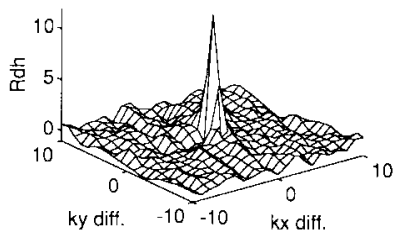

(b)

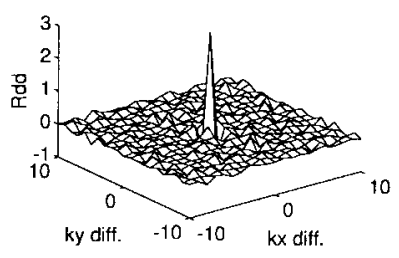

(d)
Fig. 1. Time-average autocorrelation functions of the 2-D DWT images of an $\mathrm{fBm}$ field with parameter $H=0.5$ and Haar basis for the case of $m=1$. (a) $R a[(10,10),(k x 2, k y 2)]$. (b) $\operatorname{Rdh}[(10,10),(k x 2, k y 2)]$. (c) $R d v[(10,10),(k x 2, k y 2)]$. (d) $\operatorname{Rdd}[(10,10),(k x 2, k y 2)]$.

$R_{f}\left(\vec{\tau}_{i j}\right) \equiv \mathcal{E}\left\{\boldsymbol{f}\left(\vec{r}_{i_{1} j_{1}}\right) \boldsymbol{f}^{*}\left(\vec{r}_{i_{2} j_{2}}\right)\right\}, \mu_{f} \equiv \mathcal{E}\left\{\boldsymbol{f}\left(\vec{r}_{i j}\right)\right\}$ and $\vec{\tau}_{i j} \equiv$ $\vec{r}_{i_{1} j_{1}}-\vec{r}_{i_{2} j_{2}}, i_{1}, j_{1}, i_{2}$, and $j_{2}=0,1,2, \cdots$. If $C_{f}(\overrightarrow{0})<\infty$ and $C_{f}\left(\vec{\tau}_{i j}\right) \rightarrow 0$ as $\left\|\vec{\tau}_{i j}\right\| \rightarrow \infty$, the $\boldsymbol{f}\left(\vec{r}_{i j}\right)$ is mean ergodic. Assume that $\boldsymbol{f}\left(\vec{r}_{i j}\right)$ is Gaussian; then, $\boldsymbol{f}\left(\vec{r}_{i j}\right)$ is correlation ergodic if $R_{f}\left(\vec{\tau}_{i j}\right) \rightarrow 0$ as $\left\|\vec{\tau}_{i j}\right\| \rightarrow \infty$.

Consider a zero mean sampled 2-D fBm random field with parameter $H$

$$
B\left[n_{x}, n_{y}\right]=B_{H}\left(n_{x} \Delta x, n_{y} \Delta y\right), \quad \forall n_{x}, n_{y} \in \boldsymbol{Z}
$$

where $\Delta x$ and $\Delta y$ are the sampling periods of the $x, y$ directions, respectively. From [p. 250], the autocorrelation function of the 2-D $\mathrm{fBm}$ itself $\left(B\left[n_{x}, n_{y}\right]\right)$ is written as

$$
\begin{aligned}
R_{B}\left[\left(n_{x 1}, n_{y 1}\right),\left(n_{x 2}, n_{y 2}\right)\right] & \\
= & \frac{K}{2}\left\{\left(n_{x 1}^{2}+n_{y 1}^{2}\right)^{H}+\left(n_{x 2}^{2}+n_{y 2}^{2}\right)^{H}\right. \\
& \left.-\left(\left(n_{x 1}-n_{x 2}\right)^{2}+\left(n_{y 1}-n_{y 2}\right)^{2}\right)^{H}\right\}
\end{aligned}
$$

where $K$ is a constant.

\section{B. Decorrelation Properties of The Three Detail Images of fBm Fields}

In our previous work [17], we have concluded that the three detail images of the 2-D fBm random field are all WSS. The following theorem will show further that the autocorrelation functions of the three detail images are all decorrelated.

Theorem 1: Suppose that a wavelet function has the vanishing moment $M$. Then, the autocorrelation functions of the horizontal and vertical detail images related to a $2-\mathrm{D} \mathrm{fBm}$ random field $B\left[n_{x}, n_{y}\right]$ denoted as $D_{m}^{\boldsymbol{H}} B$ and $D_{m}^{\boldsymbol{V}} B$, respectively, decay as $\mathcal{O}\left(\left(\tau_{x}^{2}+\right.\right.$ $\left.\tau_{y}^{2}\right)^{H-\nu}$ ) with $\nu \geq(M / 2)$, where $\tau_{x} \equiv n_{x 1}-n_{x 2} \neq 0$ and $\tau_{y} \equiv n_{y 1}-n_{y 2} \neq 0$ for all $n_{x 1}, n_{x 2}, n_{y 1}$ and $n_{y 2} \in Z$. Furthermore, for the diagonal detail image denoted as $D_{m}^{D} B$, the autocorrelation function decays with the order of $H-\nu, \nu \geq M$.

Proof: The proof of $D_{m}^{\boldsymbol{H}} B, D_{m}^{\boldsymbol{V}} B$ and $D_{m}^{\boldsymbol{D}} B$ are similar. Herein, we only take one of them shown below in detail. Let $\tau_{x} \equiv k_{x 1}-k_{x 2}$ and $\tau_{y} \equiv k_{y 1}-k_{y 2}$ for all $k_{x 1}, k_{x 2}, k_{y 1}, k_{y 2} \in \boldsymbol{Z}$.

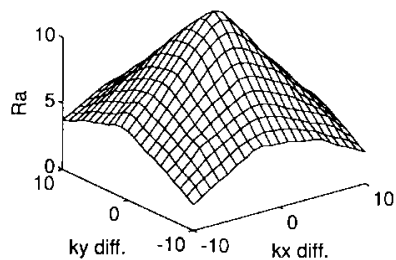

(a)

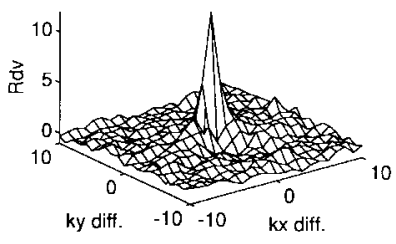

(c)

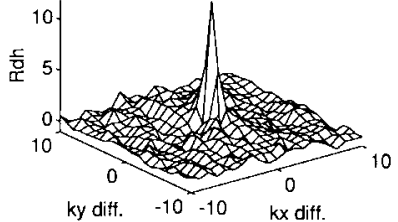

(b)

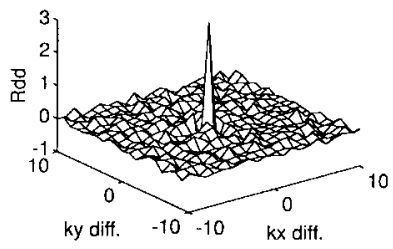

(d)
Fig. 2. Ensemble-average autocorrelation functions of the 2-D DWT images of an $\mathrm{fBm}$ field with parameter $H=0.5$ and Haar basis for the case of $m=1$. (a) $R a[(10,10),(k x 2, k y 2)]$. (b) $\operatorname{Rdh}[(10,10),(k x 2, k y 2)]$. (c) $R d v[(10,10),(k x 2, k y 2)] .(\mathrm{d}) \operatorname{Rdd}[(10,10),(k x 2, k y 2)]$.

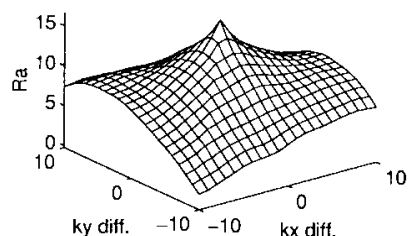

(a)

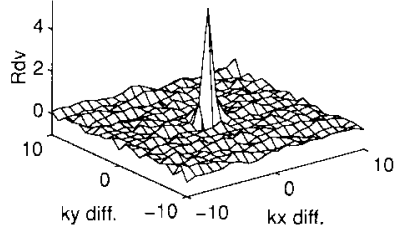

(c)

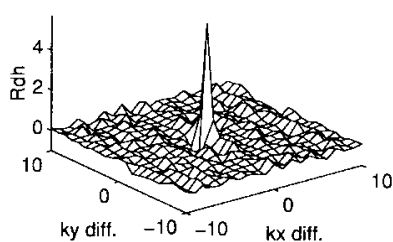

(b)

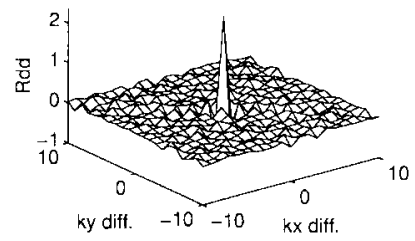

(d)
Fig. 3. Time-average autocorrelation functions of the 2-D DWT images of an $\mathrm{fBm}$ field with parameter $H=0.2$ and Daubechies 2 basis for the case of $m=1$. (a) $R a[(10,10),(k x 2, k y 2)]$. (b) $R d h[(10,10),(k x 2, k y 2)]$. (c) $R d v[(10,10),(k x 2, k y 2)]$. (d) $R d d[(10,10),(k x 2, k y 2)]$.

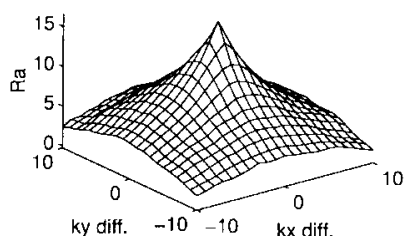

(a)

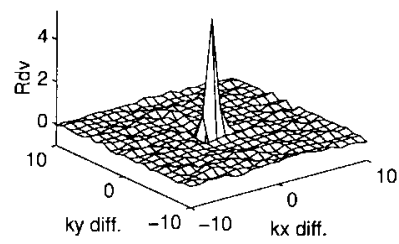

(c)

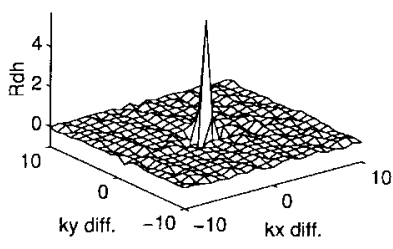

(b)

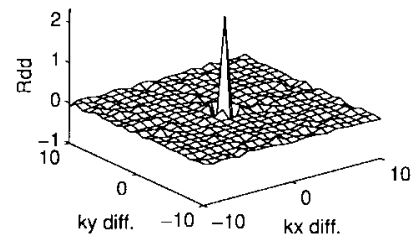

(d)
Fig. 4. Ensemble-average autocorrelation functions of the 2-D DWT images of an $\mathrm{fBm}$ field with parameter $H=0.2$ and Daubechies 2 basis for the case of $m=1$. (a) $R a[(10,10),(k x 2, k y 2)]$. (b) $\operatorname{Rdh}[(10,10),(k x 2, k y 2)]$. (c) $R d v[(10,10),(k x 2, k y 2)]$. (d) $R d d[(10,10),(k x 2, k y 2)]$. 
The autocorrelation of $D_{m}^{\boldsymbol{H}}(B)$ is written as

$$
\begin{aligned}
& R_{D} \boldsymbol{H}_{m}(B) \\
& {\left[\tau_{x}, \tau_{y}\right] \equiv } \mathcal{E}\left\{D_{m}^{\boldsymbol{H}} B\left[k_{x 1}, k_{y 1}\right] \overline{D_{m}^{\boldsymbol{H}} B\left[k_{x 2}, k_{y 2}\right]}\right\} \\
&= \Gamma^{H}\left\{\sum_{i_{1}} \cdots \sum_{i_{m}} \sum_{l_{1}} \cdots \sum_{l_{m}} \sum_{j_{1}}\right. \\
& \cdots \sum_{j_{m}} \sum_{t_{1}} \cdots \sum_{t_{m}} h\left[i_{1}\right] \\
& \cdots h\left[i_{m}\right] g\left[l_{1}\right] \cdots h\left[l_{m}\right] \\
& \times \overline{h\left[j_{1}\right]} \cdots \overline{h\left[j_{m}\right]} \overline{g\left[t_{1}\right]} \cdots \overline{h\left[t_{m}\right]} \\
&\left.\cdot\left(-\frac{K}{2}\right) P(\alpha, \beta)\right\}
\end{aligned}
$$

where $Q_{x} \equiv i_{m}+2 i_{m-1}+\cdots+2^{m-1} i_{1}-l_{m}-2 l_{m-1}-\cdots-2^{m-1} l_{1}$, and $Q_{y} \equiv j_{m}+2 j_{m-1}+\cdots+2^{m-1} j_{1}-t_{m}-2 t_{m-1}-\cdots-2^{m-1} t_{1}$. Let $\Gamma \equiv\left(2^{m} \tau_{x}\right)^{2}+\left(2^{m} \tau_{y}\right)^{2}, \alpha \equiv\left(Q_{x} / \Gamma\right), \beta \equiv\left(Q_{y} / \Gamma\right), \vec{v} \equiv$ $\left[\begin{array}{ll}Q_{x} & Q_{y}\end{array}\right]^{T}$ and $P(\alpha, \beta) \equiv\left[1+\Gamma \alpha^{2}+\Gamma \beta^{2}+2^{m+1} \tau_{x} \alpha+2^{m+1} \tau_{y} \beta\right]^{H}$. Define $\Omega(\vec{q}) \equiv\left\{r \mid \Sigma_{i=1}^{2} r_{i} / q_{i} \leq 1\right\}, \Omega_{0}(\vec{q}) \equiv\left\{r \mid \Sigma_{i=1}^{2} r_{i} / q_{i}<1\right\}$, and the differentiable closure of $\Omega_{0}(\vec{q})$ is given by $\overline{\Omega_{0}}(\vec{q}) \equiv\{r+t \mid r \in$ $\Omega_{0}(\vec{q})$ and $\left.|t| \leq 1\right\}$, where $\vec{q} \equiv\left[\begin{array}{ll}q_{1} & q_{2}\end{array}\right]^{T}, q_{1}, q_{2} \in \boldsymbol{R}^{+}$. Clearly, it is found that $P \in C^{(\infty, \infty)}$ on $\boldsymbol{R}^{2}$. The Taylor formula for real-valued $P(\alpha, \beta)$ at $(\alpha, \beta)=(0,0)$ is expressed as

$$
\begin{aligned}
P(\alpha, \beta)= & \sum_{r \in \Omega} \frac{1}{r !} D^{r} P(0,0)(\vec{v})^{r} \Gamma^{-r}+R_{(0,0)}^{\vec{q}} P\left(\vec{v} \Gamma^{-1}\right) \\
= & 1+2 H\left(2^{m} \tau_{x} Q_{x}+2^{m} \tau_{y} Q_{y}\right) \Gamma^{-1} \\
& +\sum_{r \in \Omega, r_{1}, r_{2} \neq 0,1} \frac{1}{r !} D^{r} P(0,0)(\vec{v})^{r} \Gamma^{-r} \\
& +R_{(0,0)}^{\vec{q}} P\left(\vec{v} \Gamma^{-1}\right)
\end{aligned}
$$

where $R_{(0,0)}^{\vec{q}} P\left(\vec{v} \Gamma^{-1}\right) \equiv \Sigma_{r \in \overline{\Omega_{0}} \backslash \Omega}\left(A_{r} /|r|\right) D^{r} P\left(\theta_{r} \vec{v} \Gamma^{-1}\right)\left(\vec{v} \Gamma^{-1}\right)^{r}$, $D^{r}$ denotes the partial derivative $\partial^{|r|} /(\partial \vec{v})^{r},\left\{r \in \overline{\Omega_{0}} \backslash \Omega\right\}$ represents $\left\{\left(r \in \overline{\Omega_{0}} \cup \Omega\right) \cap\left(r \notin \overline{\Omega_{0}} \cap \Omega\right)\right\}$ and $A_{r}=\Sigma\{(1 / \gamma !)$ : $\gamma \in \Omega_{0} \wedge \gamma+s=r$ for some $s$ with $\left.|s|=1\right\}$, satisfying $\theta_{r}$ $=\theta_{\gamma}$ whenever $|r|=|\gamma|$, and $\lim _{(\alpha, \beta) \rightarrow(0,0)}\left(R_{(0,0)}^{\vec{q}} P(\alpha, \beta) /|\alpha|^{q_{1}}+\right.$ $\left.|\beta|^{q_{2}}\right)=0[13$, pp. 5-8]. Then, we have

$$
\begin{aligned}
{\left[\left(Q_{x}+\right.\right.} & \left.\left.2^{m} \tau_{x}\right)^{2}+\left(Q_{y}+2^{m} \tau_{y}\right)^{2}\right]^{H} \\
= & \Gamma^{H} P(\alpha, \beta)=\Gamma^{H} \\
& \cdot\left\{\sum_{r \in \Omega} \frac{1}{r !} D^{r} P(0,0)(\vec{v})^{r} \Gamma^{-r}+R_{(0,0)}^{\vec{q}} P\left(\vec{v} \Gamma^{-1}\right)\right\} .
\end{aligned}
$$

Since $\psi$ has the vanishing moment $M$, i.e., $\int_{-\infty}^{\infty} t^{l} \psi(t)=0$, for $l=0,1,2, \cdots, M-1$ or, equivalently, $\Sigma_{k} g[k] k^{l}=0$, for $l=0,1,2, \cdots, M-1[7$, p. 142], therefore, we obtain

$$
\begin{aligned}
R_{D} \boldsymbol{H}_{(B)} & {\left[\tau_{x}, \tau_{y}\right] } \\
= & \left(\tau_{x}^{2}+\tau_{y}^{2}\right)^{H-M}\left(-\frac{K}{2}\right) 2^{2 m(H-M)} \sum_{i_{1}} \\
& \cdots \sum_{t_{m}} h\left[i_{1}\right] \cdots h\left[i_{m}\right] g\left[l_{1}\right] \cdots h\left[l_{m}\right] \overline{h\left[j_{1}\right]} \cdots \overline{h\left[j_{m}\right]} \\
& \times \overline{g\left[t_{1}\right]} \cdots \overline{h\left[t_{m}\right]} \\
& \cdot\left\{\sum_{r \in \Omega, r \geq M} \frac{1}{r !} D^{r} P(0,0)(\vec{v})^{r} 2^{-2 m(r-M)}\right. \\
& \left.\cdot\left(\tau_{x}^{2}+\tau_{y}^{2}\right)^{-(r-M)}+R_{(0,0)}^{\vec{q}} P\left(\vec{v}(\Gamma)^{-1}\right)\right\} .
\end{aligned}
$$

Because the $M$ th-order partials for $P(\alpha, \beta)$ depend on $\tau_{x}^{M}, \tau_{y}^{M}$ and $\tau_{x}^{n_{1}} \tau_{y}^{n_{2}}$, where $n_{1}+n_{2}=M$ and $n_{1}, n_{2}>0$, the $M$ th-order term of $\{\cdot\}$ in (8) is bounded below by $\Gamma^{-(M / 2)}$. Hence, $R_{D} \boldsymbol{H}_{(B)}\left[\tau_{x}, \tau_{y}\right]$

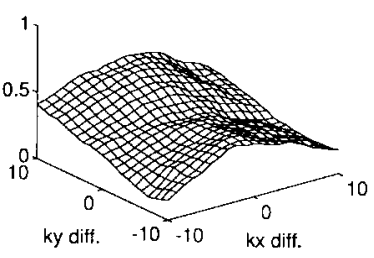

(a)

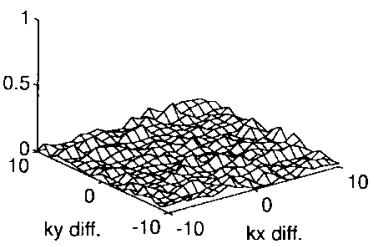

(c)

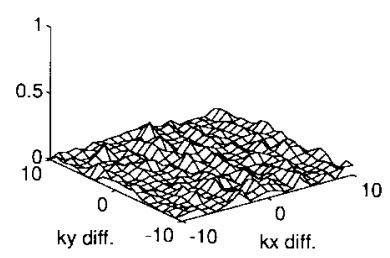

(b)

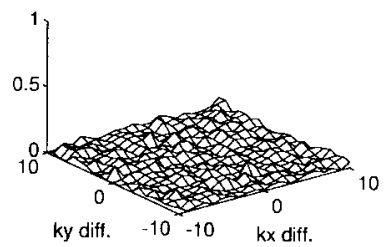

(d)
Fig. 5. Absolutely relative error between the time-average (Fig. 1) and the ensemble-average (Fig. 2). (a) A.R.E. of $R a[(10,10),(k x 2, k y 2)]$. (b) A.R.E. of $R d h[(10,10),(k x 2, k y 2)]$. (c) A.R.E. of $R d v[(10,10),(k x 2, k y 2)]$. (d) A.R.E. of $R d d[(10,10),(k x 2, k y 2)]$.

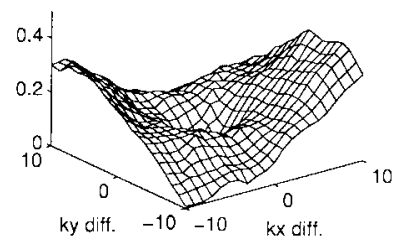

(a)

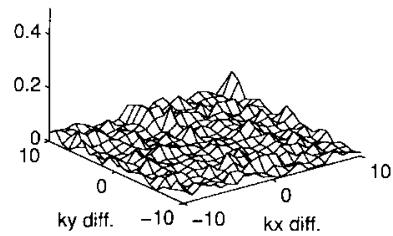

(c)

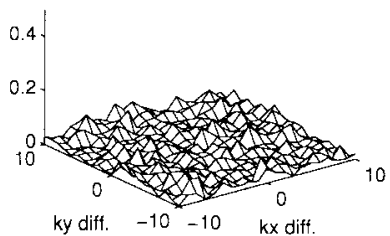

(b)

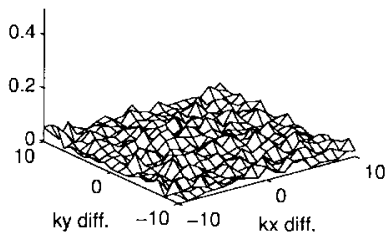

(d)
Fig. 6. Absolutely relative error between the time-average (Fig. 3) and the ensemble-average (Fig. 4). (a) A.R.E. of $R a[(10,10),(k x 2, k y 2)]$. (b) A.R.E. of $R d h[(10,10),(k x 2, k y 2)]$ (c) A.R.E. of $R d v[(10,10),(k x 2, k y 2)]$. (d) A.R.E. of $\operatorname{Rdd}[(10,10),(k x 2, k y 2)]$.

decays as $\mathcal{O}\left(\left(\tau_{x}^{2}+\tau_{y}^{2}\right)^{(H-(M / 2)}\right)$ at least.

Particularly, for the diagonal detail image case, the terms of $\tau_{x}^{n_{1}} \tau_{y}^{n_{2}}, n_{1}+n_{2}=M$ in the autocorrelation function will vanish by $g$ after the filter operation along the indexes $i_{1}, l_{1}, j_{1}$, and $t_{1}$. Therefore, the $M$ th-order term in $R_{D_{m}} D_{(B)}$ is bounded below by $\Gamma^{-M}$. Hence, $R_{D} \boldsymbol{D}_{(B)}\left[\tau_{x}, \tau_{y}\right]$ decays faster as $\mathcal{O}\left(\left(\tau_{x}^{2}+\tau_{y}^{2}\right)^{(H-M)}\right)$.

The approximation image is close to the original 2-D fBm random field. In addition, the autocorrelation function of the approximation image is near the original 2-D fBm random field and is different from the three detail images, which decay fast.

Remark 1: The decaying property of the approximation image of a 2-D fBm random field $B\left[n_{x}, n_{y}\right]$ denoted by $A_{m} B$ is not included in Theorem 1.

The autocorrelation function of $D_{m}^{\boldsymbol{H}} B$ denoted by $R_{D} \boldsymbol{H}\left[\left(k_{x 1}, k_{y 1}\right),\left(k_{x 2}, k_{y 2}\right)\right]$ is dependent only on $\tau_{x} \equiv k_{x 1}-k_{x 2}$ and $\tau_{y} \equiv k_{y 1}-k_{y 2}$, and $R_{D} \boldsymbol{H}\left[\tau_{x},-\tau_{y}\right]=R_{D} \boldsymbol{H}\left[-\tau_{x},-\tau_{y}\right]=$ $R_{D} \boldsymbol{H}\left[\tau_{x}, \tau_{y}\right]$, i.e., $R_{D} \boldsymbol{H}$ is symmetric. Therefore, in addition to Theorem 1, the detail image $D_{m}^{\boldsymbol{H}} B$ could be approximated to a white 2-D random field, which is similar to the other detail images $D_{m}^{V} B$ and $D_{m}^{D} B$. 


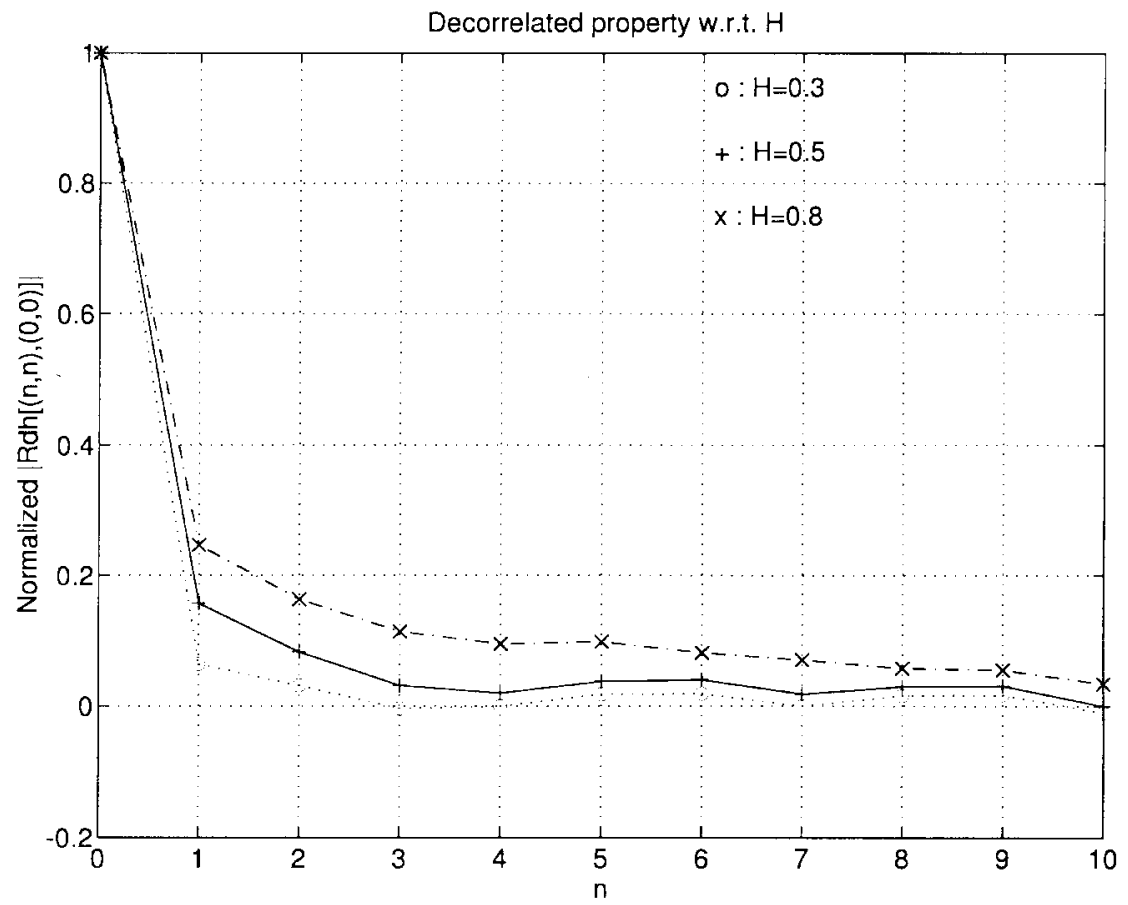

Fig. 7. Profiles of normalized $R_{D} \boldsymbol{H}$ of an fBm field with $H=0.3, H=0.5, H=0.8$ and Haar basis for $m=1$, where 'o': $H=0.3$, ' + ': $H=0.5$, 'x': $H=0.8$.

Remark 2: From Theorem 1, the three detail images of a 2-D fBm random field behave much more like white noises as the parameter $H$ is small.

\section{The Ergodicity of the 2-D DWT of fBm Fields}

Since the means of the three detail images of the fBm field are equal to zero, the corresponding covariance functions are equal to the autocorrelation functions.

Theorem 2: Let $\psi(t)$ be an orthogonal wavelet supported over the interval $\left[-K_{1}, K_{2}\right], K_{1}$ and $K_{2}>0$. The three detail images of an fBm field are defined as in (1)-(4). Then, these three detail images are mean ergodic.

Proof: Based on the results in the last subsection and Corollary 1 , it is concluded that the three detail images are mean ergodic.

Theorem 3: If an $\mathrm{fBm}$ field has Gaussian distribution, then the 2-D DWT of the fBm field is also correlation ergodic.

Proof: The reason is the same as the proof of Theorem 2.

\section{NUMERICAL EXAMPLES}

In this simulation, the spectral synthesis method in [11, pp. 96-105] is used to generate the $\mathrm{fBm}$ field denoted by $B\left[n_{x}, n_{y}\right], n_{x}, n_{y}=$ $0,1, \cdots, 127$ with parameter $H=0.5$ and $H=0.2$. It is shown numerically that the time average of the approximation image of an $\mathrm{fBm}$ field is not equal to the ensemble average of itself. However, the three detail images of the $\mathrm{fBm}$ field have the property of ergodicity, where the Haar and Daubechies $N=2$ functions are chosen as the wavelet bases, i.e., the coefficients of the Haar function are $h[0]=$ $h[1]=g[0]=-g[1]=(1 / \sqrt{2}), h[n]=g[n]=0$ for $n \neq 0,1$ and for the Daubechies $N=2$ function, $h[0]=0.48296, h[1]=$ $0.83652, h[2]=0.22414, h[3]=-0.12941$. The approximation and three detail images of the $\mathrm{fBm}$ field, which are denoted as $A_{m}\left[k_{x}, k_{y}\right]$ and $D_{m}^{\boldsymbol{\theta}}\left[k_{x}, k_{y}\right], \boldsymbol{\theta} \equiv \boldsymbol{H}, \boldsymbol{V}$ or $\boldsymbol{D}, m, k_{x}$ and $k_{y} \in \boldsymbol{Z}$, are mean zero. The corresponding autocorrelation functions for $m=1$ are calculated by the time-average (T.A.) and the ensemble-average (E.A.) methods, and the experimental results are shown in Figs. 1-4, respectively. The absolutely relative error (A.R.E.) in Figs. 5 and 6 is defined as $\left|\mathcal{E}_{\text {T.A. }}\{B\}-\mathcal{E}_{\text {E.A. }}\{B\} / \mathcal{E}_{\text {T.A. }\left[k_{x} \text { diff } .=0, k_{y} \text { diff } .=0\right]}\right|$, where the E.A. is calculated by the Monte Carlo simulation of 1280 runs. From these six figures, it is shown that the approximation image of the $\mathrm{fBm}$ field is not correlationergodic. Due to the rounding error, the T.A. values is very close to the E.A. values. Therefore, the three detail images of the $\mathrm{fBm}$ field are concluded to be correlation ergodic as shown in Theorem 3.

Meanwhile, two phenomena of the theoretical results to Theorem 1 are found as the following:

1) The autocorrelation functions of $D_{m}^{\boldsymbol{H}} B, D_{m}^{V} B$ and $D_{m}^{D} B$ decay very fast with respect to $\left[\left(k_{x 1}, k_{y 1}\right),\left(k_{x 2}, k_{y 2}\right)\right]$. See Figs. 1-4.

2) From Fig. 7, the correlation functions of the three detail images decay fast when the parameter $H$ decreases. i.e., the rate of decaying is dependent on the parameter $H$.

\section{CONCLUSION}

The ergodicity properties of random processes/fields are very important to calculate the moment values in experiments. Although the ergodicity (time homogeneous) of $\mathrm{fBm}$ processes has been taken into practical application prevailing in a lot of research, it is not recognized as ergodic if it is concluded by the ergodicity theorem. In this work, the ergodicity properties of the wavelet coefficients (1-D case) of fBm processes and the three detail images (2-D case) of fBm fields are shown. Therefore, it will provide a mathematical base for the time-average moment values of the three detail images of $\mathrm{fBm}$ fields.

\section{REFERENCES}

[1] I. Daubechies, Ten Lectures on Wavelets. Philadelphia, PA: SIAM, 1992.

[2] K. Falconer, Fractal Geometry: Mathematical Foundations and Applications. New York: Wiley, 1990. 
[3] P. Flandrin, "Wavelet analysis and synthesis of fractional Brownian motion," IEEE Trans. Inform. Theory, vol. 38, pp. 910-917, 1992.

[4] __ "On the spectrum of fractional Brownian motions," IEEE Trans. Inform. Theory, vol. 35, pp. 197-199, 1989.

[5] C. Houdré, "Wavelets, probability, and statistics: some bridges," in J. J. Benedetto and M. W. Frazier, Eds., Wavelets: Mathematics and Applications. Boca Raton, FL: CRC, 1993, ch. 9.

[6] L. M. Kaplan and C.-C. J. Kuo, "Fractal estimation from noisy data via discrete fractional Gaussian noise (DFGN) and the Haar basis," IEEE Trans. Signal Processing, vol. 41, pp. 3554-3562, Dec. 1993.

[7] T. H. Koornwinder, Ed., Wavelets: An Elementary Treatment of Theory and Applications. Singapore: World Scientific, 1993.

[8] S. G. Mallat, "A theory for multiresolution signal decomposition: The wavelet representation," IEEE Trans. Pattern Anal. Machine Intell., vol. 11, pp. 674-693, July 1989.

[9] B. B. Mandelbrot and J. W. Van Ness, "Fractional Brownian motions, fractional noises and applications," SIAM Rev., vol. 10, no. 4, pp. 422-437, 1968

[10] A. Papoulis, Probability, Random Variables, and Stochastic Processes, 2nd ed. New York: McGraw-Hill, 1984.

[11] H.-O. Peitgen and D. Saupe, Eds., The Science of Fractal Images. New York: Springer-Verlag, 1988.

[12] J. Ramanathan and O. Zeitouni, "On the wavelet transform of fractional Brownian motion," IEEE Trans. Inform. Theory, vol. 37, pp. 1156-1158, 1991.

[13] J.-A. Riestra, A Generalized Taylor's Formula for Functions of Several Variables and Certain of Its Applications. Essex, U.K.: Longman House, 1995.

[14] A. Rosenfeld and A. C. Kah, Digital Picture Processing, vol. 1, 2nd. ed. New York: Academic, 1982.

[15] H. Stark and J. W. Woods, Probability, Random Processes, and Estimation Theory for Engineers. Englewood Cliffs, NJ: Prentice-Hall, 1988.

[16] A. H. Tewfik and M. Kim, "Correlation structure of the discrete wavelet coefficients of fractional Brownian motion," IEEE Trans. Inform. The ory, vol. 38, pp. 904-909, 1992.

[17] B.-F. Wu and Y.-L. Su, "A wavelet-based approach to stationarize nonstationary two-dimensional stochastic signals," in Proc. Int. Symp. Multi-Technol. Inform. Process., Hsinchu, Taiwan, R.O.C., Dec. 16-18, 1996, pp. 277-282.

\section{Two-Dimensional System Optimal Realizations with $L_{2}$-Sensitivity Minimization}

Gang Li

\begin{abstract}
In this correspondence, an expression is derived for the error variance of transfer function of a two-dimensional (2-D) system. The optimal realization problem is then formulated by minimizing this variance with respect to all possible realizations of the system. This problem is shown to be equivalent to the minimization of a pure $L_{2}$ norm based sensitivity measure. It is shown that the problem can be solved using any standard minimization algorithm.
\end{abstract}

\section{INTRODUCTION}

The finite word length (FWL) effects have been considered to be one of the most serious problems in the actual implementation of a digital system. One of the methods to reduce these effects is to implement the system with an optimal realization that minimizes the transfer function sensitivity measure. Traditionally, the transfer

Manuscript received June 26, 1995; revised March 31, 1997. The associate editor coordinating the review of this paper and approving it for publication was Dr. Yun Q. Shi.

The author is with the School of Electrical and Electronic Engineering, Nanyang Technological University, Singapore (e-mail: egli@ntu.edu.sg).

Publisher Item Identifier S 1053-587X(98)02162-X. function sensitivity measure was defined with a mixture of $L_{1} / L_{2}$ norm for one-dimensional (1-D) systems (see, e.g., [1] and [2]). The corresponding results were extended to the two-dimensional (2-D) case by many researchers (see, e.g., [3]-[5]). This mixed $L_{1} / L_{2}$ based measure is mainly for an easy mathematical treatment and seems to lack mathematical justification. Recently, a pure $L_{2}$ based transfer function sensitivity measure was studied, and some properties of this measure were revealed in [6] and [7]. The main objective of this correspondence is to extend the $L_{2}$ sensitivity minimization problem from 1-D to 2-D.

It is true that the solution to the pure $L_{2}$ minimization problem does not have a closed form and requires more computation. This is the main drawback of using this pure $L_{2}$ sensitivity measure. The computational complexity is, however, not of concern here since this is in the design stage.

\section{Problem Formulation}

Consider a 2-D discrete linear time-invariant single input single output system (SISO) $H\left(z_{h}, z_{v}\right)$ of order $\left(n_{h}, n_{v}\right)$. This system can be represented with the Roesser state-space equations [8]

$$
\begin{aligned}
\left(\begin{array}{c}
x^{h}(i+1, j) \\
x^{v}(i, j+1)
\end{array}\right) & =\left(\begin{array}{cc}
A_{h} & A_{h v} \\
A_{v h} & A_{v}
\end{array}\right)\left(\begin{array}{l}
x^{h}(i, j) \\
x^{v}(i, j)
\end{array}\right)+\left(\begin{array}{l}
B_{h} \\
B_{v}
\end{array}\right) u(i, j) \\
& \triangleq A x(i, j)+B u(i, j) \\
y(i, j) & =\left(\begin{array}{ll}
C_{h} & \left.C_{v}\right)
\end{array}\right)\left(\begin{array}{l}
x^{h}(i, j) \\
x^{v}(i, j)
\end{array}\right)+d u(i, j) \\
& \triangleq C x(i, j)+d u(i, j)
\end{aligned}
$$

where $x^{h} \in R^{n_{h} \times 1}$ and $x^{v} \in R^{n_{v} \times 1}$ are called horizontally and vertically propagating local state vectors, respectively, and $A_{x} \in$ $R^{n_{x} \times n_{x}}, A_{x y} \in R^{n_{x} \times n_{y}}, B_{x} \in R^{n_{y} \times 1}, C_{x} \in R^{1 \times n_{x}}$ for $x, y=$ $h, v$, and $d \in R$.

$(A, B, C, d)$ is called a realization of the 2-D system $H\left(z_{h}, z_{v}\right)$, satisfying $H\left(z_{h}, z_{v}\right)=d+C\left(z_{h} I_{n_{h}} \oplus z_{v} I_{n_{v}}-A\right)^{-1} B$, where $\oplus$ denotes the direct sum of matrices. Denote $S_{H}$ as the set of all the realizations of $H\left(z_{h}, z_{v}\right)$. It is well known that $S_{H}$ is an infinite set and that if $\left(A_{0}, B_{0}, C_{0}, d\right) \in S_{H}, S_{H}$ can be characterized by

$$
A=T^{-1} A_{0} T \quad B=T^{-1} B_{0} \quad C=C_{0} T
$$

with $T=T_{h} \oplus T_{v}$, where $T_{h} \in R^{n_{h} \times n_{h}}$ and $T_{v} \in R^{n_{v} \times n_{v}}$ are any nonsingular (transformation) matrix.

\section{A. $L_{2}$ Sensitivity Measure}

Let $\left\{p_{i}\right\}$ be the set of the ideal parameters of a realization, and let $\left\{p_{i}^{*}\right\}$ be its FWL version. Assume that this realization has $N$ parameters. Denote $\left\{\Delta p_{i} \triangleq p_{i}-p_{i}^{*}\right\}$ as the corresponding parameter perturbations. With a first-order approximation, one has

$$
\begin{aligned}
\Delta H\left(z_{h}, z_{v}\right) & \triangleq H\left(z_{h}, z_{v}\right)-H^{*}\left(z_{h}, z_{v}\right) \\
& =\sum_{i=1}^{N} \frac{\partial H\left(z_{h}, z_{v}\right)}{\partial p_{i}} \Delta p_{i} .
\end{aligned}
$$

We adopt a statistical approach where the perturbations of the parameters are considered to be independent random variables uniformly distributed within the range $\left[-\frac{1}{2} 2^{-B_{c}}, \frac{1}{2} 2^{-B_{c}}\right]$ for a fixedpoint implementation of $B_{c}$ bits (see, e.g., [9]). We now define the transfer function error measure as

$$
\sigma_{H}^{2} \triangleq \frac{1}{(2 \pi)^{2}} \int_{-\pi}^{\pi} \int_{-\pi}^{\pi} E\left[\left|\Delta H\left(e^{j \omega_{h}}, e^{j \omega_{v}}\right)\right|^{2}\right] d \omega_{h} d \omega_{v}
$$

where $E(\cdot)$ denotes the ensemble average operation. 